\title{
The Berkeley tunable far infrared laser spectrometers
}

\author{
G. A. Blake, ${ }^{\text {a) }}$ K. B. Laughlin, ${ }^{\text {b) }}$ R. C. Cohen, K. L. Busarow, D.-H. Gwo, ${ }^{\text {) }}$ \\ C. A. Schmuttenmaer, D. W. Steyert, and R. J. Saykally \\ Department of Chemistry, University of California, and Materials and Chemical Sciences Division, \\ Lawrence Berkeley Laboratory, Berkeley, California 94720
}

(Received 24 September 1990; accepted for publication 10 March 1991)

\begin{abstract}
A detailed description is presented for a tunable far infrared laser spectrometer based on frequency mixing of an optically pumped molecular gas laser with tunable microwave radiation in a Schottky point contact diode. The system has been operated on over 30 laser lines in the range $10-100 \mathrm{~cm}^{-1}$ and exhibits a maximum absorption sensitivity near one part in $10^{6}$. Each laser line can be tuned by $\pm 110 \mathrm{GHz}$ with first-order sidebands. Applications of this instrument are detailed in the preceding paper.
\end{abstract}

\section{THE BERKELEY TUNABLE FAR-INFRARED LASER SPECTROMETER}

\section{A. General description}

Tunable far-infrared (FIR) lasers have become powerful tools for investigating the structures of ions, radicals, and clusters, and for probing intermolecular forces through measurement of FIR spectra of van der Waals complexes. In the preceding paper we have described the rapid evolution of FIR laser spectroscopy and some recent applications. In this article we present a detailed description of the tunable FIR laser spectrometers currently used at Berkeley. We begin with a relatively general overview of the design, and then proceed to the details of construction and operation. It is our hope that this article will serve as a useful guide to those who seek to construct similar systems.

The design of the tunable FIR laser systems used at Berkeley is similar to that of Farhoomand et al. ${ }^{1}$ In the following, we first present a general description of this design, in sufficient detail to afford all readers a reasonable understanding of the underlying principles and function. We then proceed to describe each component of the system in sufficient detail to effectively guide those actually seeking to construct a similar apparatus.

The overall experimental design is diagrammed in Fig. 1. The complete spectrometer is built on a $5 \mathrm{ft} . \times 12 \mathrm{ft}$. vibration isolation honeycomb table. $\mathrm{A} \mathrm{CO}_{2}$ laser provides an intense mid-infrared beam (maximum power $>150 \mathrm{~W}$ ) that is used to pump a molecular gas FIR laser. The $\mathrm{CO}_{2}$ laser is line tunable over some 100 different vibrationrotation transitions between 9.1 and $11.0 \mu \mathrm{m}$ using a precision grating in first-order autocollimation. The output frequency is fine-tuned over the $65 \mathrm{MHz}$ free spectral range of the cavity (limited by its $2.3 \mathrm{~m}$ length) using a piezoelectric transducer (PZT), and the zeroth-order beam reflected from the grating is focused into a $\mathrm{CO}_{2}$ spectrum analyzer to identify the laser line.

The FIR laser is pumped coaxially by the $\mathrm{CO}_{2}$ laser beam, which circulates between the FIR laser end mirrors after expanding through a $4 \mathrm{~mm}$ hole in the input coupler. The $2.5 \mathrm{~m}$ cavity of the FIR laser is of the dielectric waveguide design, with planar gold-coated copper end mirrors. FIR power is coupled out through a $10-\mathrm{mm}$-diam hole in the end mirror, which is backed by a hybrid quartz/ dielectric mirror to reflect the pump beam, while transmitting the FIR output.

The output beam of the FIR laser then enters a Martin-Puplett polarizing diplexer, which couples the laser radiation onto the (Schottky diode) corner cube mixer, while simultaneously extracting the tunable sidebands. The first polarizer (the analyzer) is rotated so the FIR laser beam (either horizontally or vertically polarized) is reflected completely. Any residual cross polarization of the laser output beam is transmitted, thus purifying the polarization. The beamsplitting polarizer is set at a $45^{\circ}$ angle projected onto the plane perpendicular to the propagation of the beam, so that the power splits equally into both arms of the diplexer. The two beams are reflected using retroreflectors mounted with one face horizontal. These devices rotate $45^{\circ}$ incident polarization by $90^{\circ}$ so that the beam initially transmitted through the beamsplitter reflects upon recombination, and vice-versa. Thus, the recombined beam is always transmitted towards the corner cube, and tuning the movable mirror cycles the polarization from vertical to horizontal, with elliptical polarization being produced at intermediate positions.

The corner cube preferentially couples to radiation polarized in the plane of the whisker antenna (see below), which is horizontal for our design. With the diplexer set to couple maximum laser power onto the diode, the reradiated, horizontally polarized beam at exactly the laser wavelength will be reflected back into the laser by the analyzing polarizer by reversibility. Unless the corner cube is perfectly aligned, however, some reradiated laser power is vertically polarized. Hence some of the sideband power is horizontally polarized. The latter can then couple into the laser cavity, causing severe baseline fluctuations when fre-

\footnotetext{
2)Division of Geological and Planetary Sciences, California Institute of Technology, MS-170-25-, Pasadena, CA 91125.

b) Research Laboratories, Rohm \& Haas Company, 727 Norristown Road, Spring House, PA 19477.

c) Department of Physics, Hansen Laboratory (GP-B, MS-4085), Stanford University, Stanford, CA 94035.
} 


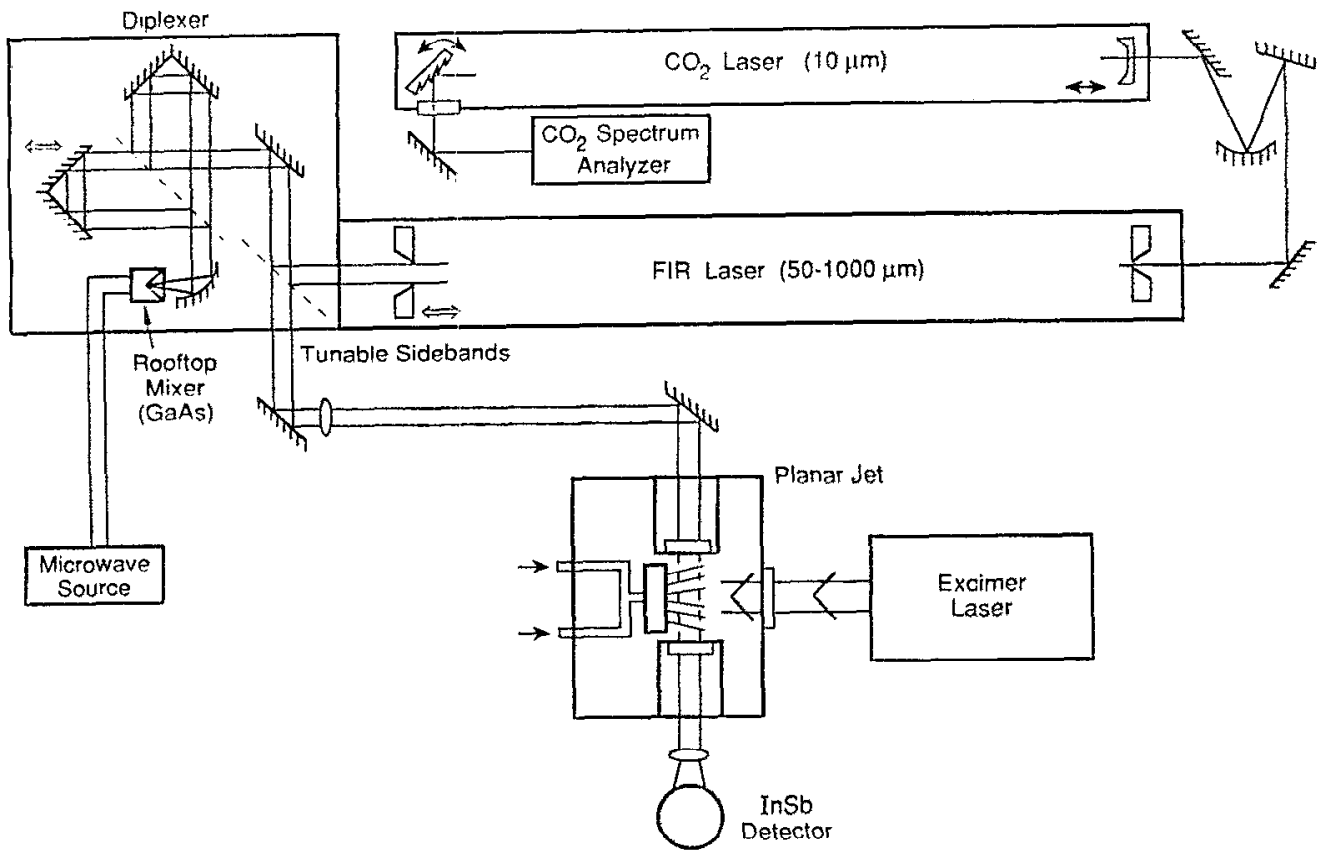

FIG. 1. Schematic diagram of the Berkeley tunable FIR laser spectrometer.

quency modulation is used. A Fabry-Perot cavity has therefore been installed between the laser and the analyzing polarizer to help eliminate feedback from the sidebands.

Because the sidebands have a different wavelength than the laser, there exists a movable retroreflector position at $\lambda_{\text {microwave }} / 4$ where both of the sidebands are polarized $90^{\circ}$ with respect to the laser, and are thus transmitted through the analyzing polarizer, provided the microwave frequency is low ( $2 \%$ ) compared to that of the laser. For higher microwave frequencies each sideband must be coupled out individually to obtain optimum power. The analyzer can be easily rotated to reflect a FIR laser beam of either vertical or horizontal polarization, facilitating conversion between different laser lines, which are essentially totally linearly polarized with a dielectric waveguide cavity.

The laser and microwave frequencies are mixed in a GaAs Schottky barrier diode, which is contacted by a metal whisker and mounted at the apex of a corner cube reflector. Frequency mixing results from the nonlinear response of the diode to the electric fields of the laser and microwave radiation. The FIR laser beam is focused at a right angle by an electroformed off-axis parabolic mirror. A rotation stage allows the corner cube to be adjusted to the optimum angle with respect to the incident beam, and the entire assembly is mounted on an $X Y Z$ translation stage. Under optimum conditions for coupling the laser beam onto the diode, sidebands are reradiated away from the corner cube with the same radiation pattern as the incoming laser beam; the focusing mirror thus collimates the outgoing beam as well.

The Berkeley corner cube design allows in situ optimization of the antenna-to-corner distance using a micrometer that translates the back reflector. The base of the cube is sloped to avoid creating an efficient retroreflector for the laser beam. Sloping the ground plane has a rather small effect on the coupling and reradiation efficiencies, ${ }^{2}$ but reduces the separation efficiency required of the diplexer.

The whisker that contacts the diode serves as the antenna for receiving and transmitting the FIR beams, and carries the necessary dc bias, as well as the microwave radiation, when coaxial coupling is used. It enters from the front of the corner reflector, angling down over the diode with appropriate length. The diode chip is soldered to a post, which is mounted to a differential micrometer for precise vertical control during contacting.

Microwave radiation may be coupled onto the diode either by a coaxial cable or with a waveguide. For the application of microwave power to the diode at frequencies that are too high for effective transmissions with coaxial cable ( $>60 \mathrm{GHz}$ ) the diode post passes through a waveguide section embedded in the body of the corner cube, with an adjustable backshort to optimize the coupling. The coupling is optimized at frequencies where microwave power is limited, and the backshort is detuned to reduce baseline drift when sufficient power is available.

The fundamental microwave source is a digital sweep oscillator, providing approximately $10 \mathrm{~mW}$ of microwave radiation from $2-26.5 \mathrm{GHz}$. This power is usually suffcient to saturate production of the first-order sidebands. The frequency range of this phase-locked source is extended to $110 \mathrm{GHz}$ with fixed-tuned millimeter wave multipliers and, when necessary, with the use of microwave amplifiers.

After the polarizing diplexer selects out the tunable FIR sidebands, they are then directed to a sample region. Transmission through the sample region is monitored with a liquid helium cooled detector. At wavelengths longer than $300 \mu \mathrm{m}$, an InSb hot electron bolometer is used, while at shorter wavelengths the detectors of choice are either a Putley mode (cyclotron resonance assisted) InSb detector 
or a Ga-doped Ge photoconductor. Detector signals are demodulated using one of several possible modulation schemes. The signal is then collected and displayed with a PDP 11/53 computer, which also controls the microwave source.

\section{B. Optically pumped FIR laser system}

\section{Background}

The optically pumped FIR laser was invented by Chang and Bridges in $1970 .^{3}$ The basic operating principle involves the generation of a population inversion in the rotational states of a gaseous molecule by transferring a substantial fraction of the population of a single ground state rotational level into a vibrationally excited rotational state. The population inversion is usually generated within the rotational manifold of the excited state, but can also occur by population depletion of the ground state. A high power infrared laser (usually either a $\mathrm{CO}_{2}$ or $\mathrm{N}_{2} \mathrm{O}$ laser) having an accidental frequency coincidence with rovibrational transitions in the FIR lasing gas is used for the optical pumping.

Since the invention of FIR lasers, many advances have been made to increase the output power. ${ }^{4-7}$ The most efficient design employs a dielectric waveguide cavity, with the $\mathrm{CO}_{2}$ laser beam circulating coaxially between the FIR laser end mirrors. After being focused through a small hole in the input coupler, the pump beam expands slowly, reflecting off of the endmirror(s) until it is extinguished from absorption by the lasing gas or the walls of the laser tube. The waveguide cavity/coaxial pumping scheme allows more efficient use of the pump laser than open cavity resonators and/or transverse pumping schemes; a waveguide laser has a smaller mode volume because the FIR radiation is confined, and thus the intensity of pump radiation for a given pump power level is higher. Moreover, pumping the FIR laser coaxially in the center of the waveguide provides higher gain for the nearly Gaussian fundamental $\mathbf{E H}_{11}$ mode, improving the mode quality as well as the output power.

Much attention has been paid to improving the output couplers used in FIR lasers. Ideally, the pump beam should be totally reflected while the FIR beam should be partially transmitted by an amount that is optimum for the laser line currently being used. Dichroic mirrors that reflect mid-IR frequencies and partially transmit FIR frequencies are hard to obtain. If a simple hole output coupler is used, similar to the input coupler, the hole must be small enough to prevent a significant amount of pump radiation from escaping. However, larger holes that couple out more FIR radiation enhance the output power. A simple hole coupler has higher loss for the fundamental $\mathrm{EH}_{11}$ mode, which can degrade the mode quality, and it has a nonadjustable fractional transmission, requiring an average fractional coupling to be chosen. A nearly ideal hole output coupler is obtained if the center hole is covered by a dielectric coated mirror, which has high reflectivity for the pump beam, while transmitting the FIR beam efficiently. Such dielectric mirrors have some reflectivity in the FIR, which can enhance the laser oscillation if the totally reflecting gold surface surrounding the hole is coated directly onto the dielectric mirror in order to maintain a well-defined phase front. We have used two types of output couplers. The first is made of copper with a quartz dielectric mirror behind it to reflect the pump beam, and the second is made of silicon with a reflective gold coating deposited directly on the surface surrounding a centered region that is left uncoated. Hole sizes from $6-10 \mathrm{~mm}$ in diameter have been used.

Other schemes have been devised to produce an output coupler that is partially reflecting over the entire surface. Gold coating a grid or an array of squares on the dielectric works very well, but only over a very small frequency range, as the reflectance of such a fixed geometry changes dramatically with wavelength. ${ }^{8}$ Considerable improvement in the design and construction of such mirrors has been reported by Densing. ' A metal mesh interferometer coupler ${ }^{10}$ and a silicon mirror interferometer, ${ }^{11}$ both of which allow in situ optimization of the fractional coupling, have been tried with moderate success. Although the uniform coupling methods tend to have superior mode quality, one disadvantage is that they are more susceptible to instabilities resulting from feedback, as reflected FIR laser power is not then forced to find its way back through a small hole to enter the laser cavity.

A drawback to the coaxial pumping geometry is the accompanying feedback of $\mathrm{CO}_{2}$ pump laser radiation back into the $\mathrm{CO}_{2}$ laser. Such feedback can cause power instabilities and therefore increased noise in the $\mathrm{CO}_{2}$ laser and FIR laser output. The simplest approach, and the one we have chosen, to eliminate feedback problems is to make the input pump beam enter slightly skewed from the FIR laser axis. The portion of the returning beam that exits the FIR laser input coupler hole then follows a slightly different path and avoids re-entering the $\mathrm{CO}_{2}$ laser. Two more sophisticated methods of avoiding feedback have been devised. One can employ an off-axis hole with slightly curved FIR cavity end mirrors; in this case the pump beam circulates off-axis for several round trips before arriving at the entrance hole again. ${ }^{12} \mathrm{~A}$ different technique uses a quarterwave plate to circularly polarize the pump beam. A stack of $\mathrm{ZnSe}$ windows at Brewster's angle is placed between the $\mathrm{CO}_{2}$ laser and the $\lambda / 4$ plate. Any reflected radiation retracing the path of the incoming beam will arrive at the Brewster stack with polarization $90^{\circ}$ to the incoming beam, and will thus be reflected. ${ }^{13}$ This technique obviously changes the FIR laser output polarization because the pump beam is not linearly polarized. ${ }^{14}$

A high level of FIR laser output power has been obtained recently by Mansfield et $a l^{15}$ and by Farhoomand and Pickett. ${ }^{16}$ Their basic design has been emulated in the FIR lasers currently used in at Berkeley. A very high power $\mathrm{CO}_{2}$ pump laser ( $>100 \mathrm{~W}$ ) is the basis for the high FIR output power. Special considerations in the FIR laser cavity design are required to safely dissipate such high pump powers. With the $119 \mu \mathrm{m} \mathrm{CH} \mathrm{CH}_{3} \mathrm{OH}$ line, cooling of the FIR laser walls and addition of $\mathrm{He}$ buffer gas to aid relaxation processes increased the output power several- 
fold. Both groups report output powers near $1 \mathrm{~W}$. A theoretical upper limit, called the Manley-Rowe condition, is placed on the output power as a function of the wavelength because each IR pump photon can produce at most one FIR photon, with an additional loss factor of $\frac{1}{2}$ because the populations of the upper and lower states are equal if the pump transition is saturated. ${ }^{7}$ The maximum FIR output at $119 \mu \mathrm{m}$ is thus $125 \mathrm{~W} \frac{1}{2}(10 \mu \mathrm{m} / 119 \mu \mathrm{m})$ or $5.3 \mathrm{~W}$, which means that the $1.25 \mathrm{~W}$ output power reported by Farhoomand and Pickett achieves 24\% of the ManleyRowe limit.

The number of known FIR laser lines grew at a dramatic pace shortly after these lasers were invented. To date, over 2000 lines from over 60 molecules (including isotopic derivatives) have been discovered. A summary of known laser lines can be found in Ref. 17.

\section{2. $\mathrm{CO}_{2}$ laser}

The $\mathrm{CO}_{2}$ pump laser used in this spectrometer (Apollo model \#150) is a commercial model with the following specifications: length: $2.5 \mathrm{~m}$; bore: $12 \mathrm{~mm}$; power: $150 \mathrm{~W}$, single line, on 10 different lines, $120 \mathrm{~W}, 50$ lines, $170 \mathrm{~W}$ at $10.59 \mu \mathrm{m}$; polarization: vertical; divergence: $3 \mathrm{mrad}$ FWHM; gas mixture: $6 \% \mathrm{CO}_{2}, 18 \% \mathrm{~N}_{2}, 76 \% \mathrm{He}$; operating pressure: 30 Torr; $\mathrm{TE}_{00}$ mode; PZT cavity length adjustment: $0-1 \mathrm{kV}$ produces $8 \mu \mathrm{m}$ travel; free spectral range: $65 \mathrm{MHz}$.

The laser has a gas recycler, which reduces laser mix consumption by a factor of 10 . A closed cycle refrigerator provides cooling to the laser tube. If desired, the ethylene glycol/water mixture can be cooled to $-30^{\circ} \mathrm{C}$ for increased output power. The $\mathrm{CO}_{2}$ laser line is monitored with a spectrum analyzer (Optics Engineering) using the zeroth-order beam, which is specularly reflected off the grating. This is a low power beam (about $2 \mathrm{~W}$ ), which can be easily detected by the fluorescent screen with proper alignment.

The $\mathrm{CO}_{2}$ laser beam is focused into the FIR laser using a concave mirror $(2 \mathrm{~m}$ radius of curvature, $1 \mathrm{~m}$ focal length). This beam, initially $7.4 \mathrm{~mm}$ in diameter (FWHM), is focused at the input coupler to a theoretical beam waist of $0.6 \mathrm{~mm}$ (FWHM). The beam expands into the FIR laser cavity such that free space propagation would make the beam waist equal to the $38 \mathrm{~mm}$ cavity diameter after $300 \mathrm{~cm}$, or 0.6 round trip passes. Because a spherical mirror only focuses well for rays close to normal incidence, a folded mirror arrangement was adopted. Mirror focusing is superior to lenses both in cost and in the power density that can be handled. The mirrors used are gold-coated copper mirrors capable of withstanding power densities of several $\mathrm{kW} / \mathrm{cm}^{2}$. The mounts that hold the mirrors in place with nylon pins are protected from heating around the edge of the mirror by aluminum irises glued to the front of the mirror mount. A removable plexiglass box is placed over all of the mirrors to keep them free of dust and to contain any dangerous stray radiation.

\section{FIR laser cavity design}

The FIR laser operates in a dielectric waveguide configuration, implying that the $38 \mathrm{~mm}$ Pyrex tube that contains the lasing gas also serves to confine the radiation within the cavity. If the waveguide were not present, the cavity would have a Fresnel number $N=a^{2} / \lambda L$ of 0.36 at a wavelength of $400 \mu \mathrm{m}$ for a mirror radius $a=19 \mathrm{~mm}$ and cavity length $L=2.5 \mathrm{~m}$. For a stable resonator, a Fresnel number $>1$ is required to minimize diffraction losses; the round trip loss would be $39 \%$ for $N=0.36$ (Ref. 18) - too large to sustain oscillation.

Laser operation in a waveguide mode was first proposed by Marcatili and Schmeltzer in $1964,{ }^{19}$ who calculated the propagation constants for the various modes of light in both dielectric and metallic waveguides. The attenuation for a perfectly straight cylindrical waveguide with smooth walls is

$$
\alpha=\left(u_{n m} / 2 \pi\right)^{2}(\lambda)^{2} a^{-3} \operatorname{Re}\left(v_{n}\right),
$$

where $u_{n m}$ is the $m$ th root of $J_{n-1}\left(u_{n m}\right)=0, \lambda=$ wavelength, $a=$ waveguide radius, $J_{n-1}(x)$ is the $n-1$ st Bessel function of first kind, and

$$
\begin{aligned}
v_{n} & =\frac{1}{2}\left(v^{2}+1\right) /\left(v^{2}-1\right)^{1 / 2} \quad \mathrm{EH}_{n m} \text { modes, } \\
& =\left(v^{2}-1\right)^{1 / 2} \mathrm{TE}_{0 m} \text { modes, } \\
& =v^{2} /\left(v^{2}-1\right)^{1 / 2} \quad \mathrm{TM}_{0 m} \text { modes, }
\end{aligned}
$$

and $v$ is the index of refraction of the waveguide material. For $v=1.5$ (glass), the $\mathrm{EH}_{11}$ mode has the lowest loss. The gain for the $\mathrm{EH}_{1 m}$ modes of a waveguide FIR laser will be higher because the linearly polarized $\mathrm{CO}_{2}$ laser preferentially pumps the FIR lasing gas in such a way as to produce linearly polarized FIR output.

A review article by Degnan ${ }^{20}$ presents some of the minimum loss resonator configurations for a waveguide laser. The simplest one, which is chosen for the Berkeley FIR lasers, is to place flat mirrors close to the ends of, or even inside of, the waveguide. There are no losses associated with this mirror configuration, as the beam reflects directly back upon itself with the same radiation pattern. Two other low loss schemes for constructing a waveguide laser oscillator are (1) spherical end mirrors at a large distance $R$ from the end of the waveguide with $R=$ radius of curvature of the mirrors, and (2) spherical mirrors at an intermediate field distance $R / 2$ from the end of the guide. A waveguide laser has a mode volume that is constant at all points within the guide, and is independent of wavelength. In addition, the surface of constant phase is planar within the guide. These features are distinctly different from open structure resonators having curved end mirrors.

The input coupler of the FIR laser has a 4-mm-diam centered hole to allow the pump radiation to enter the cavity, and the output coupler has a centered 6-10-mmdiam hole for extracting the FIR laser radiation. The losses at each end mirror for the fundamental $\mathrm{EH}_{11}$ mode can be calculated via the fractional overlap with the power distribution on the mirror, which scales approximately as $\left[J_{0}(r)\right]^{2}$, with the first zero of the Bessel function $J_{0}$ at the waveguide inner radius. For the Berkeley lasers, the 4-mm- 
diam hole has $5 \%$ loss, and the $10-\mathrm{mm}$-diam hole has $26 \%$ loss, for a roundtrip coupling of $31 \%$ for the $\mathrm{EH}_{11}$ mode. These coupling losses must be added to the waveguide losses. In the present design, the coupling losses dominate the waveguide losses until the wavelength approaches the $1000 \mu \mathrm{m}$ range. The cavity finesse

$$
F^{*}=\pi R^{1 / 2} /(1-R)
$$

can be calculated using an average reflectivity $R_{\text {ave }}=(0.75 \times 0.95)^{1 / 2}=0.84$, yielding $F^{*}=18$. The passive cavity resonances have a half power width $\Delta v=$ FSR/ $18=3.2 \mathrm{MHz}$ (HWHM) where the free spectral range $(\mathrm{FSR})=c / 2 L=60 \mathrm{MHz}$.

\section{FIR laser construction}

The FIR laser is mounted directly alongside the $\mathrm{CO}_{2}$ laser as shown in Fig. 1, with the input coupler endblock adjacent to the $\mathrm{CO}_{2}$ laser output port. The input and output couplers are each housed in a stainless steel box, supported from the table by four 2-in.-diam plexiglass rods. These rods elevate the FIR laser axis to the same height as the $\mathrm{CO}_{2}$ laser beam ( $7.75 \mathrm{in}$. from the surface of the laser table) and also serve to thermally isolate the laser from the table. The endblocks are connected to each other by four 1 1-in. diam Invar rods which are clamped to the sides of each box. The low thermal expansion coefficient of Invar $\left(1.4 \times 10^{-6} / \mathrm{C}\right)$ passively stabilizes the cavity length against temperature changes. A $38-\mathrm{mm}$-i.d. and 244-cmlong Pyrex tube forms the waveguide of the FIR laser cavity. Two supports for the laser tube are clamped to the Invar rods at evenly spaced intervals between the endblocks to prevent the laser tube from sagging.

The laser tube has a jacket with flowing ethylene glycol/water coolant for active dissipation of the high infrared pump power that strikes the wall. A closed cycle refrigerator (Neslab \#RTE-4) circulates the cooling mixture, and the wall temperature can be lowered below ambient $\left(-30^{\circ} \mathrm{C}\right.$ minimum) if desired to increase the output power. An O-ring compressed by a beveled fiange seals the laser tube to the endblock. The lids for the endblocks are machined with a square groove that seals against the smooth surface on the top sides of the box.

The input coupler endblock is a 6.5 in. cubic stainless steel box constructed from 1-in.-thick plate. The $\mathrm{CO}_{2}$ laser beam enters the vacuum through a 0.75 -in.-diam, 4-mmthick $\mathrm{ZnSe}$ window that is antireflection coated for 10.6 $\mu \mathrm{m}$ wavelength. The input coupler, which forms one end of the FIR laser cavity while simultaneously allowing the $\mathrm{CO}_{2}$ laser pump beam to enter, is a planar 2-in.-diam, 3/8in.-thick gold-coated copper mirror (CVI Laser Corp. \#HR-CU-2.0-UC, > $99 \%$ reflectance) with a $4 \mathrm{~mm}$ hole drilled in the center. The hole is beveled on the side from which the pump beam enters to scatter a badly aligned beam. To minimize diffraction losses, the input coupler is placed within about a centimeter of the end of the waveguide. With this design, the output coupler is translated to vary the cavity length, so only angular adjustment is required for the input coupler. A gimbal mount (Lansing Model 10.203) is bolted to a 1 -in.-thick platform on the bottom of the endblock, and the input coupler is held in place with a threaded brass ring.

Motorized micrometers (Oriel model \#18031 with control box model \#18000) are used to adjust the mirror angle, with an electrical feedthrough mounted on the side of the endblock to connect the control box. The cavity can thus be aligned under vacuum, and potentially leaky mechanical vacuum feedthroughs are avoided. A primary disadvantage compared to manual mechanical adjustments, however, is their inevitable failure.

Because the $\mathrm{CO}_{2}$ pump laser is very powerful, the input coupler must be cooled to preserve alignment and for more stable output power. To accomplish this, a water cooled disk is brought into good thermal contact with the input coupler by using a threaded brass ring in the gimbal mount. The center of the disk has a hole for the pump beam to pass through, and the cooling water flows through a channel in the interior of the disk. The input and output water lines must be very flexible to allow for the precise positioning of the endmirror. We have found that flexible, leak-free connections between the endblock and water cooled disk can be made with Tygon tubing by using the Swagelok inserts for connecting soft plastic tubing to metal hardware (SS-405-for 1/4 in. tubing).

The laser tube is evacuated through a $1 \frac{1}{2}$-in.-diam port welded to the input coupler endblock; it can be sealed off using a butterfly valve and a bellows valve. A liquid nitrogen trap between the laser and the mechanical pump condenses the lasing gas in order to protect the pump and hasten outgassing of the laser when switching laser gases. The trap also prevents oil from backstreaming into the laser cavity. The FIR laser gases flow into the laser via a 1/4-in.-diam stainless tube in the output coupler endblock. Three sets of needle valves and shutoff valves are available for mixing or changing gases. At a position $10 \mathrm{~cm}$ from the inside of the laser endblock, the $1 / 4$ in. tube branches to a 10 Torr capacitance manometer (MKS \#222). The large diameter pumping port is useful for pumping down the laser prior to running, but during operation the FIR laser output power is improved substantially by throttling down the valve. This is a result of decreasing the pressure drop across the laser, allowing a larger portion of the gain medium to be close to the optimum lasing pressure.

The output coupler endblock is constructed in the same manner and with the same dimensions as the input coupler endblock. One output coupler is a planar 2-in.diam, 3/8-in.-thick gold-coated copper mirror with a 10$\mathrm{mm}$-diam hole in the middle. To prevent a large fraction of the pump beam from exiting through the output hole, the mirror is backed by a 1-in.-diam dielectric coated quartz mirror (Laser Power Optics) which reflects $10 \mu \mathrm{m}$ radiation efficiently while transmitting $45 \%$ of the FIR beam. ${ }^{16}$ This design utilizes the pump radiation more efficiently while preventing damage to the output window. The FIR radiation reflected back into the laser cavity is lost, however, unless by a coincidence in wavelength it matches up in phase with the radiation reflected from the surface of the copper mirror. The other output coupler used is a $2 \mathrm{in}$. diameter $\times 1 / 4$ in. silicon disk. A gold coating covers the 


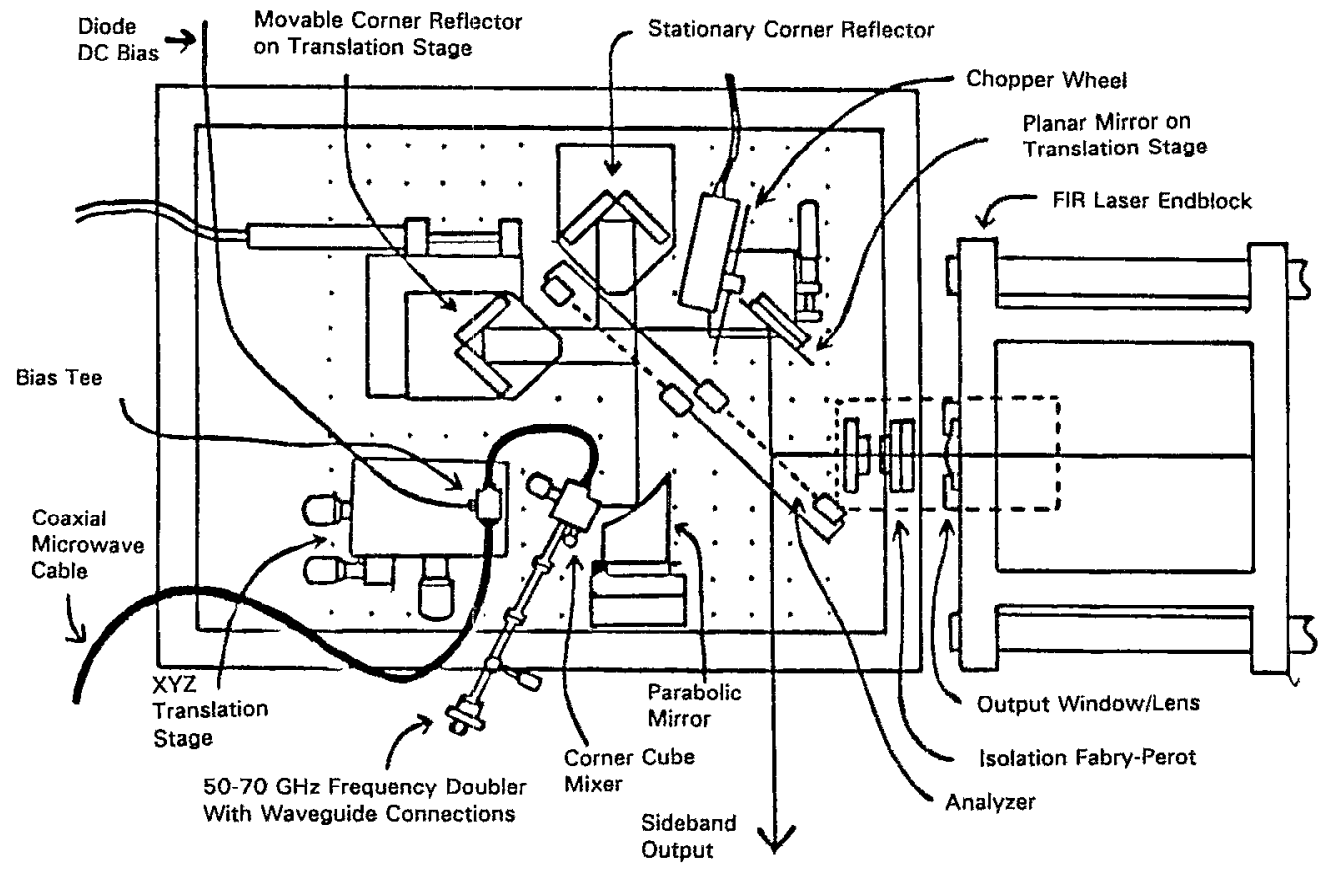

FIG. 2. Martin-Puplett polarizing diplexer. disk with the exception of $6 \mathrm{~mm}$ hole on center. The output coupler is mounted in a stainless steel holder with water circulating internally for cooling. The water lines running to the outside of the box are the same as those for the input coupler endblock. A 1 in. $Z$-cut quartz window serves as the output window and vacuum seal.

The stainless steel holder for the output coupler screws into the mounting threads of an EMT translator (MPB Technologies model \#EMT-3 with \#901 driver box). The EMT unit allows fine tuning of the cavity length, translating about $60 \mu \mathrm{m}$ over a controlling voltage from $0-42 \mathrm{~V}$. The EMT translator is mounted in a gimbal mount identical to the one at the input coupler end. Both the input and output complex are fitted with Motor Mikes to adjust the mirror angle. The gimbal on the output coupler is bolted to a translation stage (Lansing model \#20.127) for coarse adjustment of the cavity length. The minimum translation required is several orders of the longest wavelength used, or a few millimeters. This is necessary in order to sidestep coincidental overlaps in situations where more than one FIR line can oscillate simultaneously. Longer adjustments are useful for accurate wavelength measurements of laser lines of unknown frequency. The translation stage has a total range of about $12 \mathrm{~mm}$, and is driven by a Motor Mike (Oriel model \#18231) capable of encoding the position that can be read on the control box (Oriel model \# 18009). The encoder micrometer has a resolution of $0.1 \mu \mathrm{m}$.

\section{Martin-Puplett diplexer}

\section{Princlples of operation}

A detailed diagram of the Martin-Puplett polarizing interferometer is shown in Fig. 2. Polarized far-IR radiation from the laser impinges on the analyzer, which is set to totally reflect this polarization, and reflects from a movable off-axis parabola ( 14 in. F.L.) onto the beamsplitter. The beamsplitter divides the power equally into the two beams which are incident on the corner reflectors. These beams then return with their polarization rotated by $90^{\circ}$, as can be seen by analyzing the phase shifts of the components of electric field parallel and perpendicular to the surface upon reflection. Thus, the component of the incident beam that was initially reflected by the beamsplitter is transmitted upon recombination, and vice-versa. For the case of ideal optical elements, all of the incident power exits the interferometer in a direction perpendicular to the incident laser beam, and passes to the corner cube mixer. Adjusting the path length difference of the two arms with the movable corner reflector alters the relative phase of the recombined beams. The polarization of the beam exiting to the mixer cycles from horizontal to circular, to vertical, to circular (with the opposite handedness), and finally back to horizontal as the path length difference is scanned. Intermediate positions yield a beam with elliptical polarization. The period for the cycle is $\lambda_{\text {laser }} /$ pathlength difference, or $\lambda_{\text {laser }} / 2 \times$ mirror displacement. This feature is used to check the wavelength of the laser, as each maximum throughout corresponds to the same laser wavelength.

Because of the receiving properties of the corner cube, only radiation with a horizontal polarization couples onto the diode. As the movable corner reflector is translated the diode rectification cycles from a maximum value to zero and back. The reradiated power, also of horizontal polarization, re-enters the interferometer from the same direction that the laser beam entered the mixer. By reversibility of the optical path, radiation at the laser frequency exits the interferometer with the same polarization as the original FIR laser beam, and thus reflects off the analyzer and back into the FIR laser. The sidebands at $v_{\text {laser }} \pm v_{\text {MW }}$ un- 
dergo a different phase change in the interferometer, and thus arrive at the analyzer with a rotated polarization. If $L$ is the path length difference of the two arms, and $n$ and $m$ are integers, then under the conditions

$$
2 L=2 n \lambda_{\text {laser }}=(2 m+1) \lambda_{\mathrm{SB}},
$$

the sidebands will emerge with polarization perpendicular to the laser and will thus be transmitted by the analyzer. If $n=m$ for the upper sideband $\left(v_{\text {laser }}+v_{\mathrm{MW}}\right.$, the order for the sideband is one greater than for the laser), this condition is equivalent to

$$
n=\lambda_{\mathrm{MW}} / 2 \lambda_{\text {laser }}, \text { or } L=\lambda_{\mathrm{MW}} / 2,
$$

where it is assumed that

$$
\lambda_{\mathrm{SB}}=\lambda_{\text {laser }}\left[1-\left(\lambda_{\text {laser }} / \lambda_{\mathrm{MW}}\right)\right],
$$

or equivalently

$$
\lambda_{\text {laser }}<\lambda_{\mathrm{MW}}
$$

If the microwave frequency becomes comparable to the laser frequency, then the optimum condition is met less accurately and the separation efficiency of the diplexer will decrease. The nearest coincidence for the lower sideband ( $v_{\text {laser }}-v_{\mathrm{MW}}$ ) has $n=m-1$ because the order is always lower for a longer wavelength. This condition yields the same result $L=\lambda_{\mathrm{Mw}} / 2$. The displacement of the movable corner reflector from zero pathlength difference, which is read directly on the micrometer, is equal to one half the path difference $L$. For optimum coupling of sideband radiation out of the diplexer, the displacement is approximately $\lambda_{\mathrm{MW}} / 4$, set to a point where the laser power matched onto the diode is optimized. Both upper and lower sidebands are extracted with equal efficiency at this position.

\section{Diplexer construction}

The diplexer is constructed on a 16 in. $\times 20$ in. $\times 1$ in. aluminum optical plate. The plate has a square array of $\frac{1}{4}-20$ tapped holes with 1 in. spacing for mounting components, and is elevated by four plexiglass rods to match the height of the FIR laser endblocks. An O-ring groove is cut around the edge for sealing a box over the diplexer, which enables atmospheric water to be purged. The analyzer and beamsplitter are mounted parallel in a single aluminum frame placed $45^{\circ}$ to the axis of the FIR laser. A square plate cut with a hole just smaller than the o.d. of the polarizer is used to clamp each of the the polarizers in place, and can be loosened when the polarizers need to be rotated.

After initially reflecting from the analyzer, the FIR laser beam strikes an off-axis paraboloid on a rotation stage or mirror mount, which is attached to a translation stage. The mirror can be translated perpendicular to the laser axis to adjust both the position and angle at which the laser beam enters the interferometer section of the diplexer. The additional degree of freedom provided by the translation stage enables compensation for a slightly skewed laser beam. A chopper wheel is also mounted to the translation stage to monitor the beam on an oscilloscope. The motor of the chopper is encased in an aluminum box for precautionary shielding of the diode against $\mathrm{rf}$ noise radiated from the motor.

The free standing wire polarizers chosen for this experiment are available from Specac corporation (part \#57.130). They are constructed by winding a tungsten wire around a frame at a precisely controlled spacing using a lathe. The polarizers are very fragile and difficult to make, but they have the advantages of very high performance and a wide spectral range. A simpler technique for making a more rugged polarizer is to deposit metal onto a thin Mylar substrate. However, the residual reflection of the Mylar deteriorates the polarizing qualities and restricts the wavelength range; free standing wire polarizers avoid substrate reflection entirely. The polarizers are 5.25 in. in diameter with a $3.75 \mathrm{in}$. free aperture, and are constructed from $10-\mu \mathrm{m}$-diam wire with a $25 \mu \mathrm{m}$ center to center spacing. The reflection and transmission data as a function of wavelength are provided by Specac.

The beamsplitter polarizer is mounted with the wires $45^{\circ}$ from vertical in a plane perpendicular to the direction of the incoming beam. In the plane of the polarizer, this corresponds to an angle of $54.7^{\circ}$ from the vertical. For both horizontal and vertical incident polarizations, the power is split equally to both arms of the interferometer. The retrorcflectors are fabricated by Precision Lapping Co. from polished aluminum-coated glass mirrors that are glued together with the three faces perpendicular, and have a 2.5in.-wide aperture side-to-side. The retroreflectors are mounted on an aluminum plate with one face horizontal, such that the other two surfaces are vertical and the corner is symmetrically located. Only the vertical faces reflect the FIR beams; the horizontal face is not used. One of the corner reflectors is stationary, while the other is mounted to a translation stage (Lansing \#20.126, $50 \mathrm{~mm}$ travel). The translation stage is driven by an encoder mike (Oriel \# 18267) similar to the one used for the FIR laser output coupler, but with a $50 \mathrm{~mm}$ travel. The position can be read with a resolution of $0.1 \mu \mathrm{m}$ on the control box, which drives both the output coupler and diplexer Motor Mikes.

\section{Diplexer performance}

The separation efficiency of the diplexer can be deduced from the dependence of laser power leaked through the perpendicular output port on the position of the movable arm. As the movable corner reflector is translated, the leaked laser power (with no microwaves applied) varies by a factor of eight for the $432 \mu \mathrm{m}$ laser linc. For maximum laser leakage, the diplexer is set for half of the maximum power on the diode, which corresponds to transmitting a circularly polarized beam to the mixer. The reradiated beam (assumed horizontally polarized) will thus be circularly polarized upon arrival at the analyzer, for a round trip loss of four after exiting the diplexer. Thus, the separation efficiency of the diplexer is $8 \times 4=32$ at $433 \mu \mathrm{m}$.

Although the efficiency of the diplexer will be degraded by nonideal polarizer performance, the factor of 32 rejection ratio given above is substantially worse than can be explained based on the measured data for the polarizers. 
If a perfectly polarized beam enters the interferometer section of the diplexer from the mixer, then the beam that arrives at the analyzer will also be perfectly polarized if the movable arm is set properly. Nonideal performance of the polarizers will reduce the power that leaves the interferometer section by approximately $R$, or only $1 \%$ at $394 \mu \mathrm{m}$, without degrading the polarization purity. A perfectly polarized beam arriving at the analyzer will leak out the wrong port (at the laser frequency) by an amount $T_{\|}$, which is only $0.05 \%=1 / 2000$ at $394 \mu \mathrm{m}$.

Other factors are clearly more important than nonidealities in the polarizer performance in reducing the separation efficiency of the diplexer, at least at low FIR frequencies. An obvious possibility is polarization impurity in the beam that leaves the mixer. The beam at the FIR laser frequency will leak through the diplexer to the degree that the outgoing beam has a mixed polarization. Reflections from the corner cube faces are responsible for mixing the polarization, as a free antenna radiates with a pure polarization. An improvement in separation efficiency can be gained with essentially no loss in sideband power by placing anothcr polarizer between the mixer and the interferometer to purify the polarization of the reradiated beam. We have installed a third polarizer in this position and observe considerably improved performance. With proper attention to alignment at $432 \mu \mathrm{m}$ the transmitted laser is less than $10 \%$ the intensity of the sidebands.

Incomplete overlap of the beams that recombine at the beamsplitter will also reduce the diplexer performance. A residual amount of $45^{\circ}$ polarized radiation will remain at the edgc of the beam profile, $50 \%$ of which will transmit through the analyzer. Clearly, the extent of this problem depends strongly on the quality of the alignment of the diplexer, and a quantitative evaluation of this contribution to the degradation of the rejection ratio has not been made. Maintaining a larger beam diameter within the diplexer will reduce the required alignment accuracy. In addition, at low microwave frequencies, the diplexer arms must have a significantly different path length for optimum coupling of the sidebands, which will result in a poorer beam overlap due to the divergence of the beams.

\section{Isolation Fabry-Perot}

A Fabry-Perot interferometer is placed immediately after the FIR laser output window to prevent errantly coupled sideband power from re-entering the laser. The frequency scans in Fig. 3 were taken with no sample cell before the detector, and show the baseline modulations that occur when frequency modulation of the microwave source is employed. The top trace was taken with no isolating Fabry-Perot, and at the bottom it can be seen that the regular pattern of sharp spikes is eliminated with the Fabry-Perot in place. The frequency difference between the strongest peaks, $60 \mathrm{MHz}$, exactly corresponds to the free spectral range of the FIR laser. Leakage of the imperfect diplexer permits resonances of the sideband frequency with the passive FIR laser cavity to occur as the frequency is scanned, which decreases the power received by the detector. Because the finesse of the FIR laser cavity is mod-

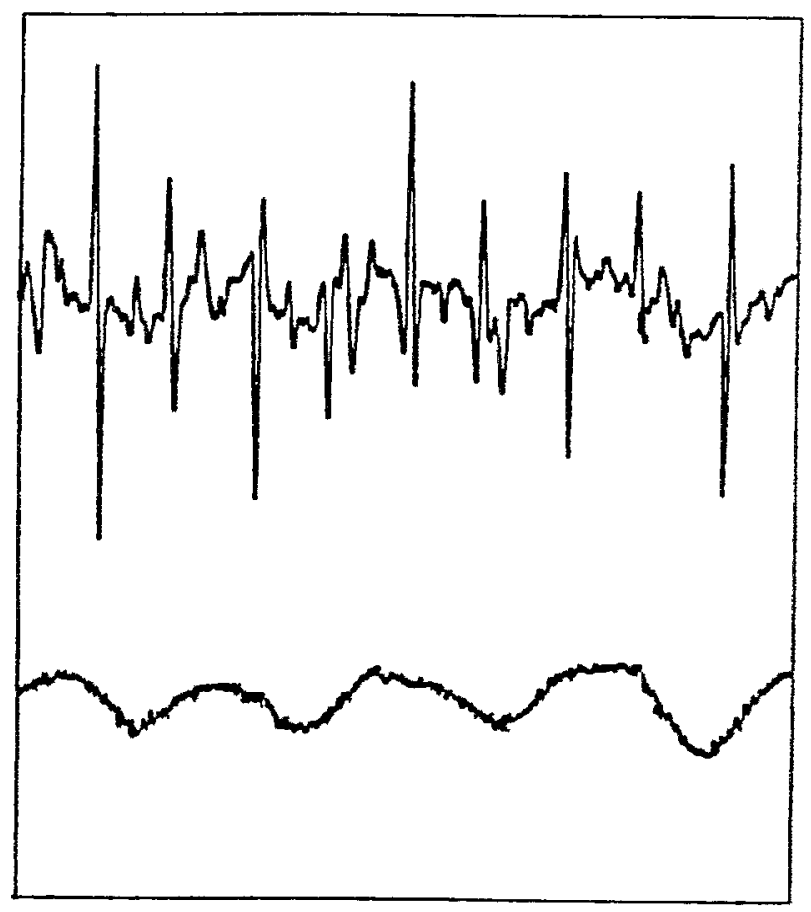

FIG. 3. Frequency modulation baseline showing resonances of sidebands with FIR laser cavity. Top trace: without isolation Fabry-Perot. Bottom trace: with Fabry-Perot. Scan width: $300 \mathrm{MHz}$. Sensitivity: $4 \mu \mathrm{V} / \mathrm{cm}$. Modulation: $2 f$ FM.

erately high $\left(F^{*}=18\right)$, the resonances are sharp $(3.2 \mathrm{MHz}$ HWHM), and thus are efficiently modulated by the FM scheme.

Ideally, when adjusted to the correct position, the diplexer should transmit all of the sidebands away from the FIR laser. Leakage resulting from the nonideality of the diplexer and large microwave frequencies relative to the FIR laser allows some of the sideband power to be reflected back into the laser. The isolation Fabry-Perot prevents such a baseline by rejecting the sidebands before they can re-enter the laser. A Fabry-Perot transmits frequencies $v=n(c / 2 L)$, where $n=$ an integer, $c=$ speed of light, and $L=$ mirror spacing. Thus if the Fabry-Perot is adjusted to transmit $v_{\text {laser }}$, the nearest frequencies transmitted are $v_{\text {laser }} \pm(c / 2 L)$. By setting the mirror spacing such that $(c / 2 L)=2 v_{\mathrm{MW}}$ (or $\left.L=\lambda_{\mathrm{MW}} / 4\right)$, the sideband frequency will be midway hetween transmission peaks, or maximally rejected. The minimum transmission for an ideal FabryPerot of finesse $F^{*}$ is $\pi^{2} / 4 F^{* 2}$, or $2 \%$ for a finesse of 11 . Interestingly, reflection of the FIR carrier from the corner reflector back into the laser cavity has no observable feedback effects.

Construction of a fairly low finesse planar surface Fabry-Perot is quite simple. Two stainless steel rings (i.d. $=22 \mathrm{~mm}$ ) are polished after machining to improve the flatness. The partially reflecting surfaces are made from free standing nickel mesh stretched tight across the rings. Nickel mesh can be purchased in a wide variety of dimensions from Buckbee-Mears Co. For best results, the mesh should be stretched twice. This is accomplished with two 
short tube sections, the smaller one fitting barely inside the larger one, and the stainless rings fitting barely inside the smaller tube. After gluing a piece of mesh to the largest tube with super glue, it is placed over the smaller tube. Super glue is applied over the rim of the smaller tube, and a moderate weight placed on top of the larger ring to stretch the mesh. A razor blade can be used to cut the large ring away after the glue has hardened. The process is then repeated placing the smaller tube over the stainless steel ring.

One of the stainless steel rings is held by a set screw to a lens mount (Newport MM-2) which has been machined to accommodate the ring. The mesh that is fastened to the mirror mount forms the stationary mirror of the FabryPerot. The other ring is also secured by a set screw to a brass ring, which is mounted to a translation stage (Newport $440-4,50 \mathrm{~mm}$ travel). A bracket for mounting the Fabry-Perot is bolted to the face of the FIR laser output coupler endblock. It has three $1 / 8$ in. precision dowel pins that match three holes in the Fabry-Perot base plate, making it easier for the Fabry-Perot to be removed and put back in the same position. The dowel pins also help avoid bumping the Fabry-Perot against the laser or diplexer when moving it. The Fabry-Perot base plate can be further secured by two $\frac{1}{4}-20$ screws.

The reflection/transmission properties of the metal mesh are a strong function of the wavelength of the radiation. A transmission curve versus the normalized wavelength is given by Ulrich and co-workers. ${ }^{10}$ At $433 \mu \mathrm{m}$, mesh having 200 lines/in. ( 80 lines $/ \mathrm{cm}$ ) has a reflectance of $75 \%$. A mesh with $75 \%$ reflectance yields a FabryPerot of finesse 11, which is sufficient for eliminating the baseline problems mentioned above. Fabry-Perots constructed from this mesh were observed to have a maximum transmission of $85 \%$. Using this Fabry-Perot, the double pass minimum transmission of the sidebands into the FIR laser and back out again is $\left(\frac{1}{64}\right)^{2}=0.02 \%$. For a high finesse Fabry-Perot, the peak transmission can be improved by gold coating the interior faces of the mesh, which reduces the absorption losses within the cavity. This would make a Fabry-Perot of a given mesh usable over a wider wavelength range.

\section{Corner cube mixer}

\section{Schottky barrier diodes}

Generation of tunable FIR sidebands is due to the nonlinear response of the diode to the electric field of the radiation. While frequency mixing is a well established technique in the radio frequency and microwave regions, it is a rather new development in the FIR. This is due mostly to the inherent frequency rolloff of the diodes commonly used for mixing. The "parasitics," which increase the conversion losses, must be reduced by advanced diode technology in order to operate diodes at FIR frequencies. Most diodes used today in the FIR are GaAs Schottky barrier diodes, where the Schottky barrier is produced by plating a layer of Pt on top of $n$-type GaAs.
Elaborate discussions of techniques for improving diode performance at high frequencies can be found in Refs. 21 and 22. Presented here are only the most basic ideas. The diode parasitics can be modeled by a simple electrical circuit which has an ideal diode placed in series with a resistance $R$ and in parallel with a capacitance $C$. The contact capacitance must be reduced to the order of $1 \mathrm{fF}$ for operation in the FIR, to ensure that the shunting impedance $1 / \omega \mathrm{C}$ is kept high $(1 / \omega \mathrm{C}=50 \Omega$ at $3 \mathrm{THz}$ for $C=1 \mathrm{fF})$. Reducing $C$ is accomplished by reducing the contact area. The diodes used in our mixer have a contact pad about $1 \mu \mathrm{m}$ in diameter. While reducing the contact area decreases the shunting capacitance, it is accompanied by an increase in the contact resistance. The diodes used at Berkeley have a dc contact resistance of about $6 \Omega$. At high frequencies new contributions to the resistance arise, and are controlled by the doping of the layers of the GaAs crystals.

The main portion of the diode is an $n^{+}$-doped GaAs substrate, with an epitaxial layer of $n$-type GaAs coated on top. This surface is covered with an insulating layer of $\mathrm{SiO}_{2}$, which is etched away using photolithography to form a square array of holes about $1 \mu \mathrm{m}$ in diameter with a few $\mu \mathrm{m}$ spacing. Platinum is then electrochemically deposited into the holes, along with a top layer of gold to improve electrical contact with the whisker. A successful diode contact results when the tip of the metal whisker enters one of the holes in the $\mathrm{SiO}_{2}$ layer and touches the gold surface. Diodes used at Berkeley were purchased from Mattauch and co-workers at the University of Virginia. The diodes presently installed in the mixer are from batch 117. Other high frequency diodes are available from Millitech Corporation (South Deerfield, MA) and Farran Technology Limited (Cork, Ireland).

These diodes require a dc bias current of $700 \mu \mathrm{A}$ for optimum sideband production. This is provided by adjustable constant-current source powered by batteries to isolate the diode from noise on the ac power lines.

\section{FIR laser coupling theory}

The original method for converting FIR radiation to current in the diode used a closed structure mixer, which incorporated waveguide input coupling similar to a harmonic generator. ${ }^{23}$ At FIR frequencies, an open structure mixer is more efficient than the waveguide mixer. With an open structure design, the whisker is not mounted within a waveguide. Rather, it is placed near the apex of a corner reflector, and the radiation is focused onto the whisker optically, employing free space propagation techniques. The crossover between open and closed structure heterodyne receiver performance has been found to occur at about $250 \mathrm{GHz},{ }^{24}$ although the authors note that very recent results indicate that improved waveguide mixers may outperform open structure mixers to frequencies as high as $350-400 \mathrm{GHz}$. A principal experimental difficulty encountered with waveguide operation at high frequencies is the extremely small dimensions required for single mode waveguides, and the increased surface resistance losses associated with the smaller volume/surface ratio. 
In an open structure mixer, the whisker that contacts the diode acts as an antenna, with a polished corner behind it to help capture the radiation. The FIR laser beam is focused optically to match the antenna pattern of the whisker/reflector combination. Krautle, Sauter, and Schultz ${ }^{25}$ first calculated the beam patterns of such a mixer, yielding the optimum structure of a whisker $4 \lambda$ long placed $1.2 \lambda$ from the corner. Their results also showed that a $90^{\circ}$ corner angle is nearly optimal. Another set of calculations demonstrated that only a very minor improvement could be obtained by using a cylindrical reflector, and resulted in a mixer that was less broadband due to a wavelength dependence of the optimum cylinder radius. ${ }^{26}$ The antenna pattern corresponding to the $90^{\circ}$ corner reflector having the whisker geometry rnentioned above is concentrated almost entirely in a single lobe directed $25^{\circ}$ from the axis of the whisker, which is very nearly cylindrically symmetric, and has a divergence angle of $16^{\circ}$ full width at the $3 \mathrm{~dB}$ points. The incident and reradiated beams are polarized in the plane that contains the corner and the whisker. Fetterman et $a l .{ }^{27}$ experimentally verified the antenna pattern for the optimum configuration, using $6 \mathrm{GHz}$ microwave radiation and a 100 times scale model.

The antenna reciprocity theorem ${ }^{25}$ dictates that the optimum whisker/reflector arrangement for coupling radiation to the diode is also best for extracting the reradiated beam at nearly the same wavelength. The diplexer described previously is ideally suited for separating the beams that double back along the same path. Calculations by Sauter, Schultz, and Wohlleben ${ }^{2}$ show that the bottom reflector plane makes a very minor contribution to the strength of the antenna lobe, and can be eliminated. Moreover, constructing a mixer without the base reflector eliminates retroreflection of the beam at the laser frequency, and thus reduces the required separation efficiency of the diplexer. Research on the optimum corner cube geometry continues with recent results from Grossman ${ }^{28}$ and Zmuidzinas, Betz, and Boreiko ${ }^{29}$ giving a more detailed theoretical analysis of the FIR coupling.

\section{Mixer construction}

In designing a mixer system for generating tunable sidebands, there were three principal goals. First, it is important that contacting of the diode with the metal whisker be convenient and straightforward. Second, the microwave radiation should efficiently couple to the diode over the frequency range of the sources used with a minimum of unwanted resonances. Last, it would be advantageous to optimize the whisker/corner reflector spacing in situ for a given FIR laser beam wavelength.

The corner cube reflector is employed to enhance the coupling of FIR radiation onto the diode antenna. The construction of the corner cube, obtained from Custom Microwave, is diagrammed in Figs. 4-6. It is constructed from two symmetric halves, each with a face polished to a mirror finish. The halves are held together by screws, with precision dowel pins maintaining the alignment. The whole corner cube, including the reflector faces, is gold plated to improve reflection and eliminate corrosion. A micrometer

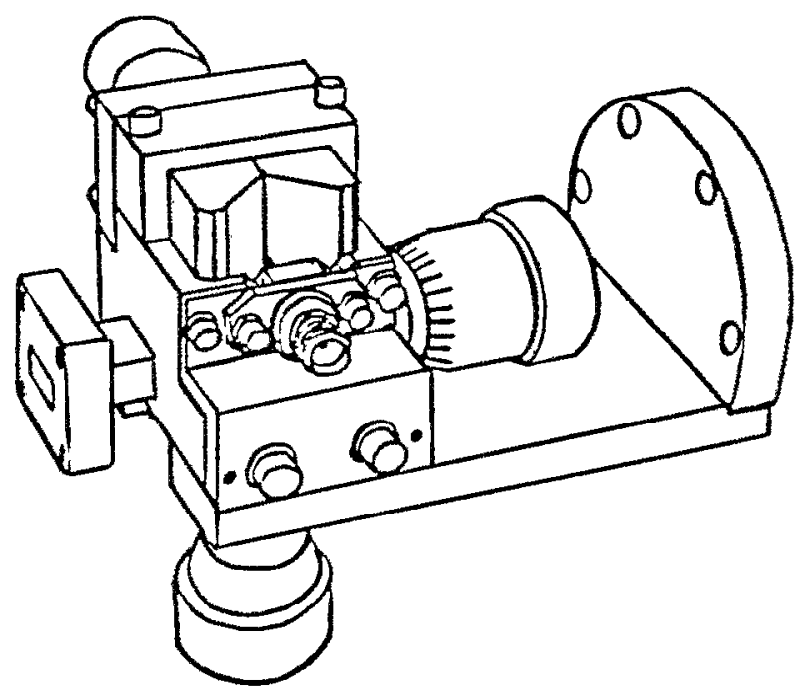

FIG. 4. Corner cube mixer with tunable back reflector and hybrid coaxial cable/waveguide microwave coupling.

drives the corner reflector in a direction perpendicular to the diode post with essentially no side-to-side motion. The body of the corner cube stops the reflector before it touches the diode post as long as the post is not bent, allowing the whisker-to-corner spacing to be optimized in situ without fear of destroying the diode contact.

The plane directly beneath the corner reflector, through which the diode post penetrates, is sloped at a $19^{\circ}$ angle with respect to the plane perpendicular to the axis of the diode post. If it were not sloped, the corner reflector would create a retroreflector and efficiently return the incident FIR laser beam. As mentioned previously, calculations show that the matching of radiation onto the diode is

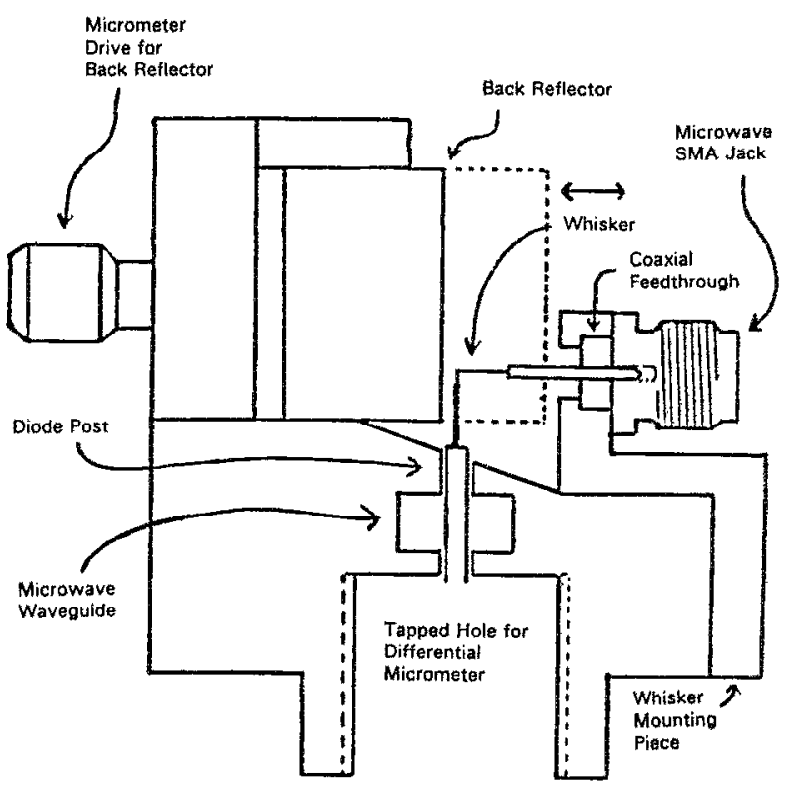

FIG. 5. Cross-section side view of corner cube mixer. 


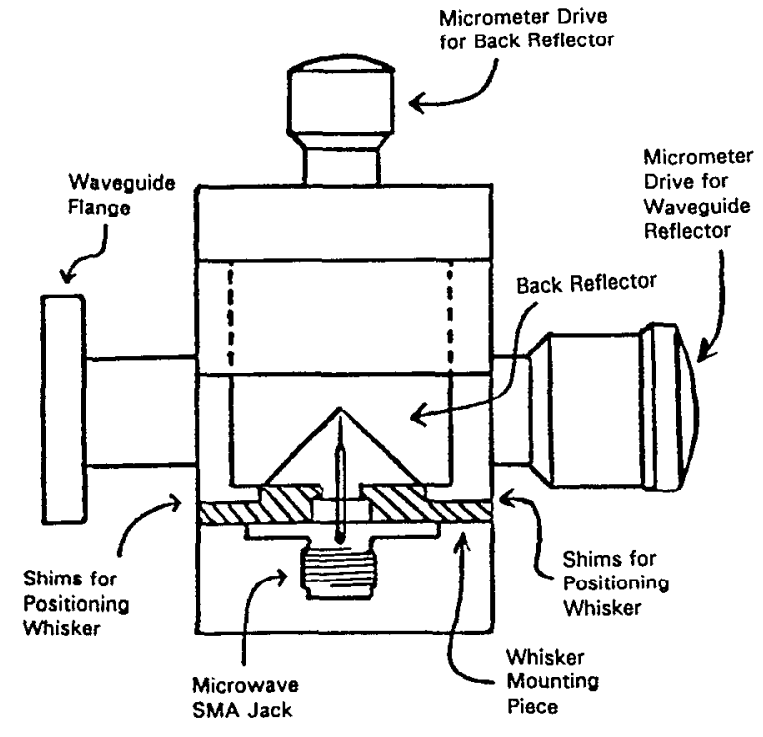

FIG. 6. Top view of corner cube mixer.

not significantly enhanced by the base plane, but it increases the required separation efficiency of the diplexer. The $19^{\circ}$ angle was chosen to ensure that an incident beam directed $25^{\circ}$ from the diode post axis will reflect back out at a very different angle.

The base of the corner cube has four \#4-40 tapped holes to attach a mounting bracket. The corner cube is suspended sideways with the whisker and corner in a horizontal plane. The top of the mounting bracket is bolted to the bottom of a rotation stage (Newport \#RSA-1), using a Plexiglass adapter to electrically isolate the mixer. Because the contact point is on the axis containing the center of the rotation stage, the whisker changes angle but does not translate when the stage is adjusted. A 6 in. $\times 2.75$ in. $\times 0.5$ in. aluminum plate with a hole for the Plexiglass adapter is used to fasten the rotation stage to a $X Y Z$ translation stage (Newport \#466). This compact translation stage allows fine positioning of the whisker with $2 \mathrm{~mm}$ range of travel in each direction.

Each diode is soldered to a gold plated brass post. The diode posts have a very thin top section 0.015 in. diameter $\times 0.175 \mathrm{in}$. length, which allows the corner reflector to be positioned very close to the diode contact point, and a much thicker base ( 0.060 in. diameter), which is mounted in the differential micrometer. Because the corner reflector must be very close to the contact point for short wavelengths, the diodes are soldered near the edge of the post, allowing the diode to be rotated to the back. The diode posts are held by a set screw in a differential micrometer, and can be easily replaced when a new diode crystal is required. The differential micrometer unit screws into the base of the corner cube by a variable amount for coarse adjustment of the vertical position of the diode. The coarse height is controlled by a threaded locking ring that clamps onto the outer threads of the differential micrometer unit. Adjusting the position of the threaded ring changes the height of the diode as well as its orientation as the threaded ring tightens against the base of the corner cube.

After the differential micrometer unit is secured in the corner cube, the very fine and smooth control (with no rotation of the diode post) provided by the differential micrometer is used for contacting the diode.

\section{Whiskers and coaxial microwave coupling}

The whiskers used to contact the diode are of 0.001 in.-diam $\mathrm{Au} / \mathrm{Ni}(82 \% / 18 \%)$ alloy wire (California Fine Wire Company). Phosphor bronze alloy was used previously, but the new material is less susceptible to corrosion, etches to a sharp point more easily, and is somewhat harder. The whiskers are soldered to the tip of a coaxial feedthrough (EMC part \#HS-80-0), which is constructed from a brass pin embedded in a glass disk that has a metal layer on the outside. The opposite side of the feedthrough fits into an SMA microwave jack (EMC part \#6520) which is bolted onto the whisker mounting piece by two \#2-56 screws. The glass disk of the coaxial feedthrough fits into a matching hole in the whisker mounting piece on the front of the corner cube, and is held in place by two \#0-80 set screws. The whisker enters from opposite the corner reflector and angles down over the diode at a distance of $6.3 \mathrm{~mm}$ from the face of the feedthrough mount. By allowing the coaxial cable to connect very close to the diode contact point, the microwave coupling efficiency is improved and made more constant with respect to frequency. The power available directly from the microwave source $(10 \mathrm{dBm})$ is sufficient to saturate the diode up to 20 $\mathrm{GHz}$.

The length of the antenna that accepts the FIR radiation is defined by the distance from the $90^{\circ}$ angle to the contact point. It can be easily controlled by etching the whisker to proper length. Small reductions in length that occur when the whisker is resharpened are negligible compared to the total length of the whisker.

The whiskers are bent at a position where they extend slightly past the diode before angling down. Precise positioning of the whisker is achieved by placing various thicknesses of brass shimstock between the whisker mounting piece and the body of the corner cube, as shown in Fig. 6 . This draws the whiskers back to the desired position. The whisker also must be centered with respect to the corner reflector before a contact is made. Some left-right adjustment can be achieved by using different thicknesses of shimstock on the two sides of the whisker mounting piece, angling the whisker. Usually this is sufficient to accurately position the whisker, but when more adjustment is necessary, the whisker can be bent with fine tweezers. This takes considerable practice, and once positioned, the whisker should be left for at least an hour to relieve hysteresis before contacting. Otherwise, a good contact can be lost as the whisker drifts slowly back in the direction of its previous position.

A bias tee (Wiltron \#K250) allows both the microwaves and the dc bias current to be applied through the coaxial microwave conncetion. 


\section{Whisker etching}

To contact the diode, it is necessary that the tip of the whisker fit into a hole approximately $1 \mu \mathrm{m}$ in diameter in the diode surface. A whisker that is too blunt will be prevented from contacting by the insulating $\mathrm{SiO}_{2}$ layer on top of the crystal. To form such a sharp point, the tip of the whisker is electrochemically etched.

The etching solution, $10 \% \mathrm{KCN}$ and $5 \% \mathrm{Fe}(\mathrm{CN})_{6}$ in water, is filled nearly to the top edge of a cuvette having flat sides for easy viewing. A stainless steel welding wire is used for an electrode, and runs down the corner of the cuvette into the solution. The electrode in solution is connected to the negative end of a regulated DC power supply, while the pin vise is connected to the positive end and grounded. A mirror is placed at about a $45^{\circ}$ angle in front of the cuvette to observe the whisker with a microscope during the etching process.

When initially etching a whisker, it must be shortened to the appropriate length. With the whisker dipped into solution just short of the proper: length, a potential of about $10 \mathrm{~V}$ from a regulated dc supply is applied until the portion of the whisker below the surface dissolves away. Obtaining a properly etched whisker takes some practice. With the power supply turned off, the tip is immersed to a depth of about two or three whisker diameters. A potential of 12 to $20 \mathrm{~V}$ is turned on until the whisker etches out of solution and no more bubbling is observed. The bubbling should be fast but not rapid enough to knock the tip around. A high power microscope (Bausch and Lomb, 430 magnification) is used to check the tip for shape and sharpness. Ideally, it should look like a cone or slightly convex, and about as sharp as can be seen with the rnicroscope. A blunt tip will not contact the diode, and a cone with concave curvature will bend over under the stress of the contact.

\section{Waveguide microwave coupling}

Besides the SMA connector mentioned previously, the corner cube has a waveguide for introducing higher frequency microwave radiation to the diode. The limit of coaxial microwave cable technology is constantly being pushed to higher frequency. Currently, coaxial cable components using SMA compatible connectors are fairly standard, with high performance available at frequencies up to about $55 \mathrm{GHz}$, but Wiltron Corp. has introduced special V-type connectors with high performance above $60 \mathrm{GHz}$. At higher frequencies, determined by the current level of technology, microwave radiation is more efficiently propagated using waveguides rather than coaxial cable.

The large diameter section of the diode post fits through the center of the waveguide, which is embedded in the body of the corner cube. The inner dimensions of the waveguide (type RG-99u) are 0.061 in. $\times 0.122$ in. at the input flange, which is the proper size for microwave frequencies of $60-90 \mathrm{GHz}$. The waveguide is then tuned to provide a $50 \Omega$ termination for the radiation. A back reflector driven by a micrometer fits inside the waveguide on the opposite side from the input flange. It can be tuned to generate a standing wave with a maximum at the diode post to enhance coupling of microwaves to the diode, but is often set off resonance when sufficient microwave power is available, for more convenient frequency sweeping.

\section{E. Microwave sources}

By employing state-of-the-art frequency synthesis technology, continuously tunable microwave radiation with sufficient power to generate FIR sidebands is generated over the range $2-110 \mathrm{GHz}$. The fundamental microwave power for generating tunable sidebands is produced by a digital synthesized signal generator (Hewlett-Packard model $8673 \mathrm{~B}$ ). It provides power from $2-26.5 \mathrm{GHz}$, with the power level, and the scanning range and rate specified by push buttons on the front or by computer. The source scans digitally, with each point in the scan phase-locked to extremely high frequency accuracy. The output power is controlled by variable and step attenuators, with either internal or external leveling. Frequency modulation, amplitude modulation, and pulsing can all be performed using $\mathrm{BNC}$ inputs on the front panel.

Microwaves from the fundamental source are transmitted to the corner cube by coaxial cable. A bias tee (Wiltron) is used to couple the microwave power and dc bias current onto the same cable. The bias tee has a blocking capacitor on the microwave side to prevent de bias current from flowing to the microwave source, and an inductor on the bias side to avoid loss of microwave power. It is specified to operate from 2 to $55 \mathrm{GHz}$, but it is actually used at higher frequencies. The bias tee is bolted with plastic screws to the aluminum plate that attaches the rotation stage to the $x y z$ stage, enabling microwave and dc bias connections to be made without moving the whisker while electrically isolating the bias tee. Semirigid microwave cable is used to connect the output of the bias tee to the SMA jack on the corner cube.

Additional coverage is obtained by frequency multiplying the output of the microwave source. In doing so, all of the frequency accuracy, computer control, convenience, and modulation capabilities of the fundamental microwave source are retained. A semirigid cable is connected from the output of the microwave source to the SMA jack on a microwave doubler (Spacek model $\mathrm{Ka}-2 \mathrm{X}$ ). The frequency doubler accepts an input frequency of $13-20 \mathrm{GHz}$ at a maximum power $21.5 \mathrm{dBm}$. The $26-40 \mathrm{GHz}$ output has a conversion loss of $12.0 \mathrm{~dB}$, for a maximum output power of $9.5 \mathrm{dBm}$. This is sufficient to saturate the diode but is not adequate for driving additional multipliers.

To achieve frequencies over $40 \mathrm{GHz}$, the frequency doubler is bolted to the input flange of a ferrite isolator (Sperry \# D41V5). The output flange of the ferrite isolator is bolted to the input of a traveling wavetube amplifier (Hughes Aircraft Corp. model \#8001H12). The TWT is specified to provide $30 \mathrm{~dB}$ of gain with a minimum output power of $1 \mathrm{~W}$ over the full $26-40 \mathrm{GHz}$ range.

The TWT output passes through another ferrite isolator, then into a waveguide variable attenuator $(\mathrm{M} / \mathrm{A} \mathrm{Com}$ \#3-28-101), and to a 26-40 GHz waveguide to coax convertor (Wiltron). The noise from the TWT amplifier is reduced when operated near maximum output, and this 
requires that the waveguide variable attenuator be nearly completely closed and that a $10 \mathrm{~dB}$ fixed coaxial attenuator be used in line as well.

Much greater extension of the microwave scanning range is obtained by doubling or tripling the output of the TWT, allowing coverage of the $50-170 \mathrm{GHz}$ range. The microwave connections are identical to the frequency doubling setup described above, except that the coaxial cable is disconnected from the bias $T$ and connected to the high frequency doubler (Honeywell \# V2200N), or tripler (Millitech FTT-10). The output from the multiplier doubler exits through a $50-75 \mathrm{GHz}$ microwave flange, and is connected via a tapered waveguide adapter (Custom Microwave) to the corner cube flange. An E-H tuner (50-75 $\mathrm{GHz}$ ) is placed between the doubler and the adapter to improve coupling of microwaves onto the diode. A similar scheme is used for the triplers. The maximum power out of the doubler and tripler is small $(2.0 \mathrm{dBm}$ minimum, 5.0 $\mathrm{dBm}$ typical for a $20 \mathrm{dBm}$ input), but adequate to match onto the diode.

\section{F. Detectors}

Despite the inherent difficulties posed by the detection of low photon energy far infrared radiation, and the large background from room temperature blackbody emission spectrum, increasingly sensitive devices are now becoming available.

In the following section we give a brief overview of the device physics for the detectors used in our lab, but we concentrate on their performance as currently implemented in our laser system. We refer the reader interested in more comprehensive studies of far infrared detection to the reviews of the field by Brown and Tannenwald ${ }^{30}$ and Richards and Greenberg, ${ }^{31}$ as well as to the work of Watson and Huffman ${ }^{32}$ describing advances in construction of arrays of $\mathrm{Ge}: \mathrm{Ga}$ photoconductors.

\section{InSb hot electron bolometer and the InSb cyclotron resonance assisted (Putley mode) detectors}

In zero magnetic field, the properties of $n$-InSb at liquid $\mathrm{He}$ temperatures can be described as those of an electron gas. Far infrared radiation is absorbed by these electrons, increasing their thermal energy. This absorption is strongest below $10 \mathrm{~cm}^{-1}$, and rolls off as $1 / \lambda$ above this frequency. The absolute responsivity (detector signal divided by incident power) of several InSb detectors has been measured by Brown and Tannenwald ${ }^{30}$ to be in the range of $0.5-4.0 \mathrm{mV} / \mu \mathrm{W}$. The detector noise in this device is dominated by thermal Johnson noise

$$
V_{n}=(4 k T R B)^{1 / 2} \text {, }
$$

where $T$ is the bath temperature, $R$ the detector resistance, $B$ the measurement bandwidth, and $k$ Boltzmann's constant. For typical InSb detectors at optimum conditions, the resistance is about $3 \mathrm{k} \Omega$, giving a noise contribution of $V_{n}=0.8 \mathrm{nV}$, which corresponds to a maximum attainable NEP of $4 \times 10^{-13} \mathrm{~W} / \sqrt{\mathrm{Hz}}$. Imperfect preamplifiers and less than optimum coupling of radiation onto the detector will result in an actual performance that is less than ideal. The InSb detector in use in our lab is a commercial device with a specified electrical responsivity of $2.4 \mathrm{mV} / \mu \mathrm{W}$, and an electrical NEP of $1 \times 10^{-12} \mathrm{~W} / \sqrt{\mathrm{Hz}}$. We assume an absolute responsivity of $2 \mathrm{mV} / \mu \mathrm{W}$ in the discussion below.

The detector element itself is an $n$-InSb crystal mounted on the cold surface of a standard Infrared Labs liquid helium Dewar. The detector is mounted in a sidelooking configuration with field of view baffling on the cold surface. A conical feedhorn $(f / 2,15 \mathrm{~mm}$ aperture) serves to collect the light and focus it onto the detector. The detector is protected from unwanted radiation in the near IR by a blackpaper filter. The signal from the detector is amplified by a fixed gain $(\times 600)$ preamplifier with an input noise $V n<1 \mathrm{nV} / \sqrt{\mathrm{Hz}}$. A slight improvement in detector performance could thus be achieved with a quieter preamplifier.

In addition to the free electron absorption in $n$-InSb, there are also strong conduction and impurity level cyclotron resonance absorptions in the presence of a magnetic field. The selection rules for cyclotron resonance allow absorption for +1 helicity (counterclockwise) circularly polarized photons propagating parallel to the magnetic field; for linearly polarized light propagating perpendicular to the magnetic field the electric field of the radiation must also be perpendicular to the field. ${ }^{33}$ The optimum geometry for absorption of linearly polarized light is known as the Voight configuration.

The low frequency performance of the hot electron bolometer can be extended to at least $60 \mathrm{~cm}^{-1}$ with magnetic fields ranging to $8 \mathrm{k}$ Gauss. The absolute NEP of a Putley mode detector is optimized by choosing a field strengths such that the cyclotron resonance absorptions peak at the frequency of interest. Brown and co-worker's ${ }^{33}$ report optimized NEPs which decrease by about 2 from $20-30 \mathrm{~cm}^{-1}$, and are then flat to $50 \mathrm{~cm}^{-1}$, where they begin to rise sharply as a result of increased noise due to impact ionization.

We have constructed two Putley mode detectors. The first consists of an $n$-InSb crystal and associated light collecting optics mounted in the Voight configuration between the poles of a superconducting split-pair solenoid. The field is continuously adjustable from $0-15 \mathrm{k}$ Gauss. The electronics associated with the superconducting magnet result in a device that is physically cumbersome. As a result we have also constructed a fixed field detector using $5 \mathrm{kG}$ permanent magnets instead of a split pair solenoid. The performance of these detectors is quite similar to that obtained by Brown and co-workers. ${ }^{33}$

\section{Ge:Ga photoconductors}

Ge:Ga photoconductors with noise equivalent powers four orders of magnitude $\left(2.4 \times 10^{-17} \mathrm{~W} / \sqrt{\mathrm{Hz}}\right)$ lower than InSb detectors have been reported by Wang et al. ${ }^{34}$ Conventional $\mathrm{Ge}: \mathrm{Ga}$ photoconductors respond from 90 $250 \mathrm{~cm}^{-1}$, while stressed Ge:Ga detectors operate to frequencies as low as $50 \mathrm{~cm}^{-1}$. These detectors were utilized under conditions of extremely low photon flux and have 
TABLE I. A listing of the laser lines that have been used in the Berkeley tunable far infrared systems.

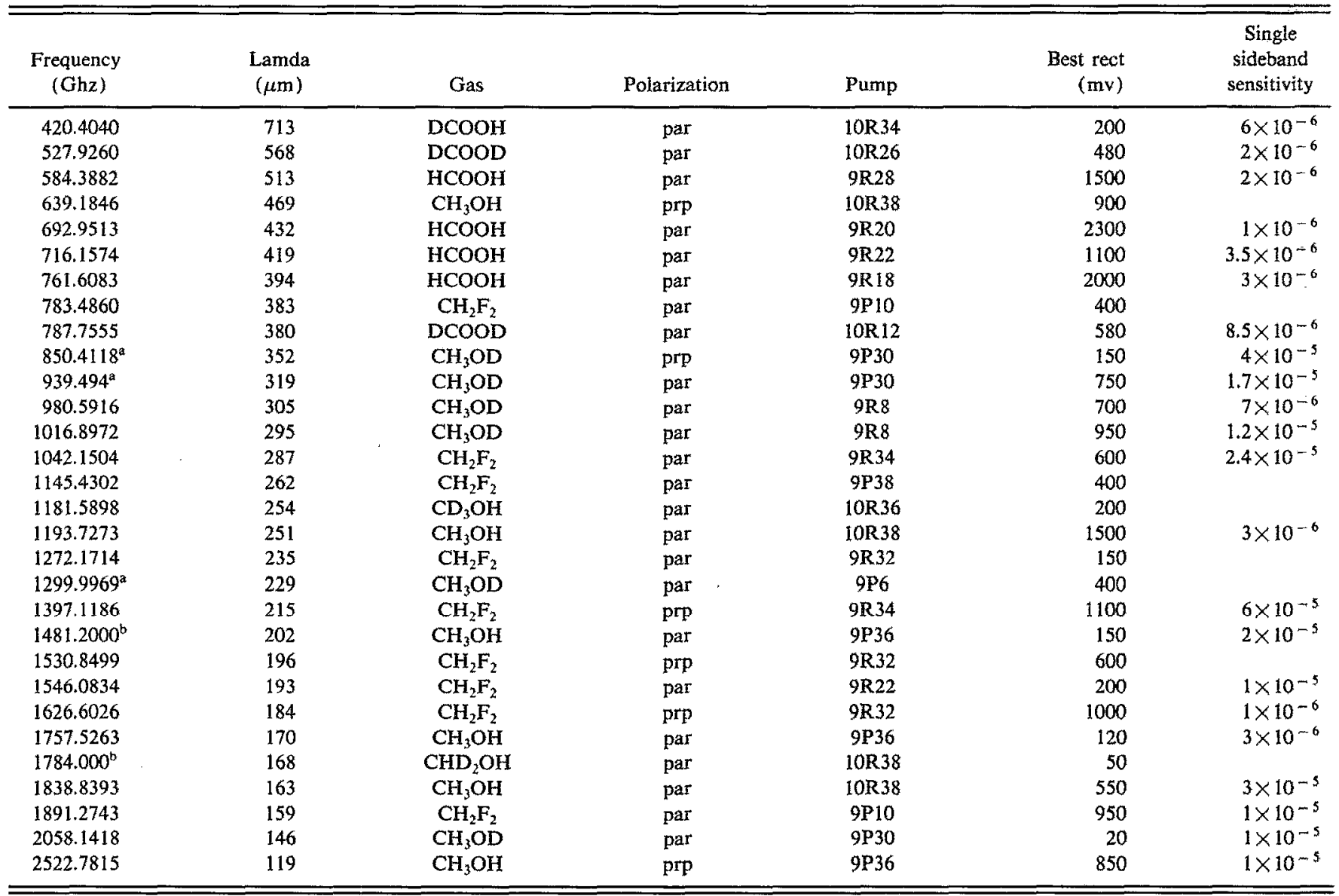

${ }^{a}$ Measured in this laboratory.

${ }^{b}$ Accurate frequencies for these lines are not currently available.

been used primarily to detect an increase in photon flux when an instrument is scanned across an astronomical emission. In the TFIR experiment, we are interested in detecting small fluctuations in the large background of laser power on the detector. In principle one should be able to achieve shot noise limited performance with these photoconductors, although such performance has not yet been demonstrated for a direct absorption experiment.

Unstressed (conventional) Ge:Ga photoconductors are available commercially from Infrared Labs. The detector used in our lab is mounted in a side-looking configuration on the cold surface of a liquid helium Dewar. A set of filters is placed in front of the detector on the $4 \mathrm{~K}$ surface to cut off unwanted blackbody radiation at wavelengths shorter than those of interest. Signal from the detector is amplified using a transimpedance amplifier (IR Labs LN8 ). We have also similarly constructed a stressed $\mathrm{Ga}: \mathrm{Ge}$ photoconductor detector which was used in our study of $\mathrm{C}_{3}{ }^{35}$ Although the NEP which is theoretically attainable with these detectors should allow shot noise limited sensitivities of 1 part in $10^{8}$, we have not yet obtained sensitivities greater than 1 part in $10^{6}$. We are currently attempting to ascertain the source of this discrepancy. Details of the results and a more comprehensive discussion of the use of
Ga:Ge photoconductors will be published in subsequent articles.

\section{SPECTROMETER PERFORMANCE}

\section{A. Sensitivity}

As described above, the sensitivity of a tunable FIR laser spectrometer is generally determined by the responsivity and noise characteristics of the detectors, since the radiation power levels available greatly exceed the blackbody background, but are normally insufficient to cause source noise to become important. Therefore, the spectrometer sensitivity varies directly with the FIR sideband power available. In Table I is given a summary of the FIR laser lines used with the Berkeley spectrometer and their performance characteristics. Not all of the results are obtainable with a single FIR output coupler, or detector. The table is a compilation of results obtained over several years with several different diodes. Many of the numbers in it can be improved substantially.

If the FIR and $\mathrm{CO}_{2}$ lasers are tuned correctly, the noise on the lock-in amplifier should be about $0.9 \mu \mathrm{V} /$ $\mathrm{Hz}^{1 / 2}$ (the InSb detector noise limit) even for very strong sideband production (several voits). The sensitivity of the 
spectrometer can be calculated by the ratio of the detector noise to the single sideband power. In the simplest case, the lock-in converts an ac signal to a dc signal, while the noise retains its rms value throughout the signal processing; thus, a factor of 2 is lost in sensitivity. The sensitivity is further reduced because full amplitude modulation of the signal is not, in general, attainable. This factor is about $\frac{1}{3}$ for an FM deviation about equal to the absorption line HWHM. It was determined by measuring a line with sufficient intensity (about 10\% absorption) to be seen easily using AM spectroscopy, enabling the absolute absorption strength to be measured directly. The calculated minimum detectable fractional absorption, neglecting baseline problems, is thus:

$$
\begin{aligned}
{\left[\frac{\Delta P}{P}\right]_{\text {min. det. }}=} & {\left[\frac{0.9 \mu \mathrm{V} / \mathrm{Hz}^{1 / 2}}{\mathrm{~V}_{\mathrm{SB}}}\right] \times[2 \times 2 \times 3] } \\
= & {\left[\frac{\text { det. noise }}{\mathrm{SB} \text { vid. sig. }}\right] \times[(\text { sing. SB conv. })} \\
& \times(\mathrm{ac} \rightarrow \mathrm{dc} \text { conv. }) \times(\text { ind. mod. })] .
\end{aligned}
$$

Two volts of sidebands therefore correspond to a sensitivity in terms of a fractional absorption of $0.6 \times 10^{-5}$ with a 1 $\mathrm{Hz}$ detection bandwidth. The absorption sensitivity corresponding to some commonly used laser lines is shown in Table I.

The sensitivity figures given in Table I correspond to measured laser power to detector noise (at a modulation frequency of $100 \mathrm{kHz}$ ), assuming that detector noise constitutes the fundamental limit. As described earlier, the recent development of long wavelength doped photoconductive mixers demands that a fully quantum analysis of the laser shot noise be performed in order to establish the ultimate sensitivity limits of the FIR laser system. The laser shot noise is a fundamental noise limit that cannot be transcended without the use of squeezed states produced via four wave mixing, and we use this noise figure as an ultimate estimate of spectrometer sensitivity.

If we define $\Delta \delta_{\min }=\Delta P / P$ as the minimum detectable absorption, then it may be shown that the shot noise limit for this quantity is given by

$$
\Delta \delta_{\min }=2.8 \times 10^{-10}\left(\nu / \eta M^{2} P_{0} \tau\right)^{1 / 2},
$$

where $v$ is the laser frequency $\left(\mathrm{cm}^{-1}\right), \eta$ is the detector quantum efficiency, $M$ is the modulation index of the detection scheme $(0<M<1), P_{0}$ is the laser power $(\mu \mathrm{W})$, and $\tau$ is the integration time. ${ }^{31}$ The increase in the minimum detectable absorption with frequency at constant power may be most easily explained by the fact that fewer photons are available for detection, and since the shot noise limit is a statistical one, fewer photons imply larger relative noise. At a wavelength of $200 \mu \mathrm{m}(1500 \mathrm{GHz})$ with FIR sideband power level of $1 \mu \mathrm{W}$, and a detector quantum efficiency of 0.25 , a modulation index of 0.3 , and an integration time of one second, we find that the quantum mechanically-limited sensitivity is $\Delta \delta_{\min }=1 \times 10^{-8}$. By reference to Table $I$, we see that several orders of magnitude improvement in sensitivity can be realized by using the state of the art detectors described in the preceding sections.

\section{B. Resolution}

The linewidth of the radiation source is determined by the stability of the FIR laser, as the contribution from the microwave source ( $<10 \mathrm{~Hz}$ FWHM) is completely negligible. Measurements indicate that the $433 \mu \mathrm{m}$ HCOOH laser line has a bandwidth of less than $100 \mathrm{kHz} F W H M$, while the $496 \mu \mathrm{m} \mathrm{CH} \mathrm{CH}_{3} \mathrm{~F}$ line was somewhat broader at $200-300 \mathrm{kHz}$ FWHM. ${ }^{24}$ These numbers could be improved by frequency locking the laser system, but the above level of resolution is sufficiently high that the spectrometer itself is unlikely to be the limiting source of resolution in most applications.

For example, for a medium weight molecule such as $\mathrm{CO}$, at $700 \mathrm{GHz}$ absorption frequency, the Doppler width is $0.83 \mathrm{MHz}$ (FWHM) at $77 \mathrm{~K}$, and $1.6 \mathrm{MHz}$ at $300 \mathrm{~K}$. The total linewidth is a convolution of Doppler broadening and pressure broadening, where the latter can be made negligible by operating at a sufficiently low pressure. Thus, the resolution of the spectrometer is usually determined by the intrinsic linewidth of the absorbing molecules. However, the planar jet supersonic expansion source developed at Berkeley by Busarow et al. ${ }^{36}$ has a much narrower linewidth than an ordinary Doppler profile. In this source, the molecules are cooled to temperatures of a few degrees Kelvin by collisions as they expand out the nozzle. The linewidths observed in the planar jet are typically about $300-400 \mathrm{kHz}$ (FWHM), so it is quite possible that source broadening is contributing substantially to, or even dominating, the observed linewidths under these conditions.

\section{Frequency stability}

The frequency precision of a measured spectral transition is affected, not only by the linewidth of the absorption, but also by drift in the FIR laser frequency. To calibrate the frcquency of an unknown line, a scan through an absorption from a reference molecule is taken immediately after scanning through the unknown transition. Uncertainties in the FIR laser frequency cancel when the two scans are compared, to the extent that there is no change over the duration of the scans. An evaluation of the drift rate of the FIR laser was made by repeated scanning through narrow, high signal-to-noise absorptions from the planar jet expansion ( $\mathrm{HCl}$ dimer) ${ }^{37}$ Although there was no active locking of the lasers, they were allowed to stabilize by warming up for a few hours. The up and down scan was $30 \mathrm{MHz}$ wide in each direction, and lasted $17 \mathrm{~s}$. The time between scanning through the hyperfine peaks discussed below was 0.6 $\mathrm{s}$, and a $30 \mathrm{mS}$ lock-in time constant was used for detection. After fitting the line profiles with a computer, the standard deviation of the peak positions of three successive scans varied by only $3 \mathrm{kHz}$. Stability over a somewhat shorter term was evaluated based on the splittings from nuclear hyperfine structure observed in various spectral 
lines. For a splitting with average value $2.208 \mathrm{MHz}$, the standard deviation was $3 \mathrm{kHz}$, the same as the line position uncertainty.

In principle, improvements in both ultimate resolution and frequency stability could be realized if the FIR laser frequency were actively stabilized over a long period of time by using a feedback circuit to position the cavity length for optimum power. However, this approach has several drawbacks. First, FIR laser frequency drift is caused not only by changes in the FIR laser cavity length, but also by changes in the $\mathrm{CO}_{2}$ laser pump frequency, and the $\mathrm{CO}_{2}$ laser must be stabilized as well. Second, and more importantly, the positions of peak power for both the FIR laser and $\mathrm{CO}_{2}$ laser cavity lengths are not the desired operating points. Both lasers must be detuned somewhat from the optimum power output for stable operation, as a result of the inevitable feedback that occurs with perfect alignments. Also the optimum frequency for absorption by the lasing gas in the FIR laser does not, in general, coincide with the peak of the $\mathrm{CO}_{2}$ gain curve, and is actually often displaced substantially.

\section{ACKNOWLEDGMENTS}

The Berkeley tunable FIR laser project has been funded by several different agencies over the ten-year development period. These include the National Science Foundation Grant \#CHE-9044712; the Director, Office of Energy Research, Office of Basic Energy Sciences, Chemical Sciences Division of the U.S. Department of Energy under Contract No. DE-AC03-76SF00098; the Army Research Office Grant \#DAAL03-86-G-0184; the Office of Naval Research Grant \#N00014-90-J-1368; and NASA Grant \#NAGW 1022. We thank these agencies for their support.

'J. Farhoomand, G. A. Blake, M. A. Frerking, and H. M. Pickett, J. Appl. Phys. 57, 1763 (1985).

${ }^{2}$ E. Sauter, G. V. Schultz, and R. Wollleben, Int. J. Infrared Millimeter Waves 5, 451 (1984).

${ }^{3}$ T. Y. Chang and T. J. Bridges, Opt. Commun. 1, 423 (1970).

${ }^{4}$ D. T. Hodges, F. B. Foote, and R. D. Reel, Appl. Phys. Lett. 29, 662 (1976).

${ }^{5}$ D. T. Hodges, F. B. Foote, and R. D. Reel, IEEE J. Quantum Electron. QE-13, 491 (1976).

${ }^{6}$ G. A. Koepf, H. R. Fetterman, and N. McAvoy, Int. J. Infrared Millimeter Waves 1, 597 (1980).

${ }^{7}$ D. T. Hodges, Infrared Phys. 18, 375 (1978).
${ }^{8}$ E. J. Danielwicz, T. K. Plant, and T. A. DeTemple, Opt. Commun. 13, 366 (1975).

${ }^{9} \mathrm{R}$. Densing, Ph.D. Thesis, Universitat Bonn, 1988.

${ }^{10}$ R. Ulrich, T. J. Bridges, and M. A. Pollack, Appl. Opt. 9, 2511 (1970).

"F. Julien and J. M. Lourtioz, Opt. Commun. 38, 294 (1981).

${ }^{12}$ G. A. Koepf and N. McAvoy, IEEE J. Quantum Electron. QE-13, 418 (1977).

${ }^{13}$ D. K. Mansfield, A. Semet, and L. C. Johnson, Appl. Phys. Lett. 37, $688(1980)$.

${ }^{14}$ D. K. Mansfield, G. J. Tesauro, L. C. Johnson, and A. Semet, Opt. Lett. 6, 230 (1981)

${ }^{15}$ D. K. Mansfield, E. Horlbeck, C. L. Bennett, and R. Chouinard, Int. J. Infrared Millimeter Waves 6, 867 (1985).

${ }^{16} \mathrm{~J}$. Farhoomand and H. M. Pickett, Int. J. Infrared Millimeter Waves 8 , 441 (1987).

${ }^{17}$ M. Ignuscio, G. Moruzzi, K. M. Evenson, and D. A. Jennings, J. Appl. Phys. 60, R161 (1986).

${ }^{18}$ A. G. Fox and T. Li, Bell Syst. Tech. J. 40, 489 (1961).

${ }^{19}$ E. A. J. Marcatili and R. A. Schmeltzer, Bell. Syst. Tech. J. 43, 1783 (1964).

${ }^{20}$ J. J. Degnan, Appl. Phys. 11, 1 (1976).

${ }^{21}$ B. J. Clifton, IREE Trans. Microwave Theory Tech. MTT-25, 457 (1977); G. T. Wrixon and W. M. Kelly, Infrared Phys. 18, 413 (1978).

${ }^{22} \mathrm{~T}$. W. Crowe, Int. J. Infrared Millimeter Waves 10, 765 (1989); L. K. Seidel and T. W. Crowe, Int. J. Infrared Millimeter Waves 10, 779 (1989).

${ }^{23}$ D. D. Bicanic, B. F. J. Zuidberg, and A. Dymanus, Appl. Phys. Lett. 32, 367 (1978).

${ }^{24}$ H. P. Roser, E. J. Durwen, R. Wattenbach, and G. V. Schultz, Int. J. Infrared Millimeter Waves 5, 301 (1984).

${ }^{25}$ H. Krautle, E. Sauter, and G. V. Schultz, Infrared Phys. 17, 477 (1977).

${ }^{26} \mathrm{H}$. Krautle, E. Sauter, and G. V. Schultz, Infrared Phys. 18, 705 (1978).

${ }^{27}$ H. R. Fetterman, P. E. Tannenwald, B. J. Clifton, W. D. Fitzgerald, and N. R. Eríckson, Appl. Phys. Lett. 33, 151 (1978).

${ }^{28}$ E. N. Grossman, Infrared Phys. 29, 875 (1989).

${ }^{29} \mathrm{~J}$. Zmuidzinas, A. L. Betz, and R. T. Boreiko, Infrared Phys. 29, 119 (1989).

${ }^{30}$ E. R. Brown and P. E. Tannenwald, Soc. Photo-Optical Instrum. Eng. 666, 38 (1986).

${ }^{31}$ P. L. Richards and L. T. Greenberg, Infrared Millimeter Waves 6, 149 (1982).

${ }^{32}$ D. M. Watson and J. E. Huffman, Appl. Phys. Lett. 52, 1602 (1988).

${ }^{33}$ E. R. Brown, M. J. Wengler, and T. G. Phillips, J. Appl. Phys. 58, 2051 (1985).

${ }^{34}$ J. Q. Wang, P. L. Richards, J. W. Beeman, N. M. Haegel, and E. E. Haller, Appl. Opt. 25, 4127 (1986).

${ }^{35}$ C. A. Schmuttenmaer, R. C. Cohen, N. Pugliano, J. R. Heath, A. L. Cooksy, K. L. Busarow, and R. J. Saykally, Science 249, 895 (1990).

${ }^{36}$ K. L. Busarow, G. A. Blake, K. B. Laughlin, R. C. Cohen, Y. T. Lee, and R. J. Saykally, Chem. Phys. Lett. 141, 289 (1987); K. L. Busarow, G. A. Blake, K. B. Laughlin, R. C. Cohen, Y. T. Lee, and R. J. Saykally, J. Chem. Phys. 89, 1268 (1988).

${ }^{37}$ G. Dlake, K. L. Busarow, R. C. Cohen, K. B. Laughlin, Y. T. Lee, and R. J. Saykally, J. Chem. Phys. 89, 6577 (1988). 